\begin{tabular}{|c|c|c|c|c|c|}
\hline MUNIBE Antropologia-Arkeologia & $n^{\circ} 65$ & $25-36$ & DONOSTIA & 2014 & ISSN 1132-2217 • eISSN 2172-4555 \\
\hline
\end{tabular}

\title{
Candil de ciervo decorado magdaleniense del yacimiento de Santa Catalina (Bizkaia, España). Tecnología y funcionalidad
}

\section{Decorated magdalenian upper part of a deer antler from the Santa Catalina site (Bizkaia, Spain). Technology and functionality}

KEY WORDS: Arte mobiliar, grabado, Magdaleniense superior, País Vasco.

PALABRAS CLAVES: Portable art, engraving, Upper Magdalenian, Basque Country.

GAKO-HITZAK: Arte higigarria, grabatua, Goi Madeleine-aldia, Euskal Herria.

Eduardo BERGANZA(1) y Rosa RUIZ IDARRAGA(1)

\section{ABSTRACT}

In this work we present a decorated upper part of a deer antler found in the Upper Magdalenian Level III, in Santa Catalina Cave (Lekeitio, Bizkaia). It is studied technologically and its engraved representations are analysed.

An outstanding portable art object was found in Level III in the archaeological deposit in Santa Catalina (Bizkaia, Spain), and is attributed to the upper Magdalenian. AMS radiocarbon dates of 12,345 \pm 85 BP $(14,007-14,945$ cal BP) and 12,425 \pm 90 BP $(14,093-15,024$ cal BP $)$ were obtained for the base of this level.

The material used to make the object was a red deer antler point, whose natural surface has not been conserved. The end opposite the point is rounded, and next to the end the antler is cut back in a concave shape. The decoration occupies both sides of the object. On one side a whole figure of a red deer hind is represented, while the other side displays two series of curved, inclined and parallel grooves. This second motif, although it appears to be non-figurative, is reminiscent of some schematic representations of deer antlers found both on portable art objects and in parietal art.

The technological study was able to reproduce the work carried out to prepare the object. The antler surface displays unorganised marks suggesting that the natural surface was removed by cutting it with a flint blade. The same implement may have been used to create the concave shape at one end of the object. Finally, the end was rounded by rubbing it on a limestone cobble.

From the functional point of view, the traces it displays and the little weight of the object mean that it cannot have been used as a working tool. The presence of some marks on the part that has been cut back suggest this part was used to haft the object or in some system of suspension. All together, the detailed results of this study seem to indicate that it should be interpreted as a prestige object or that it had a ritual use.

It is a unique artefact because of its shape and its careful decoration. The most similar objects known are two items from the Pyrenean cave of La Vache (France).

\section{RESUMEN}

En este trabajo presentamos un candil de ciervo decorado recuperado en el nivel III, Magdaleniense superior, de la cueva de Santa Catalina (Lekeitio, Bizkaia). Hacemos su estudio tecnológico y el análisis de las representaciones grabadas.

\section{LABURPENA}

Lan honetan ikusi egin daiteke Lekeitioko (Bizkaia) Santa Catalina koban aurkitutako orein adarretako puntari bruuzko teknologia-ikerketa eta grabatutako irudien ikerkuntza. Punta hori III. mailan, Goi Madeleine-aldikoan, aurkitu da.

\section{1.- INTRODUCCIÓN}

En el curso de las excavaciones del yacimiento de Santa Catalina (Lekeitio, Bizkaia) se encontró un fragmento de candil de ciervo decorado en sus dos caras. En una de ellas se identifica el contorno grabado de una figura de cierva y en la otra un motivo de líneas incisas, en apariencia, no figurativo. El soporte de este objeto tuvo una preparación que modificó su forma y su superficie. Para el análisis de esta pieza hemos llevado a cabo un estudio tecnológico a partir de un programa experimental. De esta forma se trataron de resolver aspectos tanto del diseño de la decoración como de los

(1) AOZTA eduardoberganza@irakasle.net 
de su fabricación y uso. Así mismo, hemos buscado semejanzas tecno-tipológicas en objetos aparecidos en otros yacimientos y paralelos a los motivos decorativos en representaciones similares del arte prehistórico.

La pieza se inventarió como SC.A8.139.159 y se depositó en el Euskal Museoa de Bilbao.

Creemos que con este estudio damos a conocer una pieza de excepcional importancia, que enriquece la reducida colección de arte mueble conocida hasta la fecha. Planteamos una metodología de análisis que permite reconocer su fabricación y su funcionalidad.

\section{CONTEXTO ARQUEOLÓGICO}

La cueva de Santa Catalina se localiza en el municipio de Lekeitio y se ubica en la misma línea de costa. La excavación de su yacimiento ha permitido descubrir una secuencia de tres niveles arqueológicos que se atribuyen a etapas que van desde el Magdaleniense superior hasta el Aziliense.

El objeto que ahora estudiamos se recuperó en el nivel III, Magdaleniense superior. Este nivel es muy fértil desde el punto de vista arqueológico y se caracteriza por una abundante industria lítica en la que predomina el utillaje de laminillas con dorso, una industria ósea con una numerosa colección de arpones de formas muy variadas y una interesante serie de piezas de arte mueble con decoración figurativa y geométrica.

La base del nivel en que se recuperó la pieza ha sido datada en el $12.345 \pm 85 \mathrm{BP}(14.007-14.945$ calBP $)$ y el $12.425 \pm 90$ BP (14.093-15.024 calBP) (BERGANZA et alii, 2012).

El asta decorada que estudiamos apareció en dos fragmentos. Tanto la forma de la fractura como su disposición en el relleno hacen pensar en una rotura postdeposicional. Su superficie presentaba algunas pequeñas concreciones calizas que recubrían parte de la decoración. (Fig. 1).
En el Euskal Museoa de Bilbao se procedió a su limpieza eliminando las concreciones mencionadas, así como a la unión y reintegración de ambos fragmentos.

\section{DESCRIPCIÓN DE LA PIEZA}

La pieza está realizada en un candil de ciervo. Tiene unas dimensiones máximas de 200,0 por 21,3 $\mathrm{mm}$. A efectos descriptivos hemos denominado zona distal la parte apuntada del soporte y zona proximal la opuesta; cuerpo central, a la parte del soporte entre ambos extremos y, por último, consideramos como cara A la decorada con la figura de cierva y como cara B la opuesta (Fig. 2 y 3 ).

La sección del soporte es circular en su mayor parte salvo en la más cercana al extremo proximal en que adopta una forma oval. Este extremo se ha modificado para obtener una superficie convexa con aristas redondeadas mientras el distal mantiene el aspecto natural del asta.

La zona cercana a la parte proximal está rebajada adoptando una forma cóncava respecto a la superficie natural del asta. Es lo que denominamos tramo rebajado. Su longitud es de 61,2 $\mathrm{mm}$ y su profundidad de 3 $\mathrm{mm}$. El grosor de la sección del objeto, a la altura de este rebaje, es de 15,22 $\mathrm{mm}$.

En la cara A se representa el contorno casi completo de una figura de cierva. En la cabeza destacan dos orejas, un ojo, dos pequeños ollares y se aprecia la boca con alguna dificultad. Del contorno del cuerpo se representa la línea dorso-lumbar, el pecho, la línea ventral, las cuatro patas y la cola. La figura tiene una perspectiva torcida: las orejas y ollares se sitúan de frente al observador, mientras que la cabeza y el resto de la figura están posicionados de perfil. Junto a ella se dispone un motivo formado por tres incisiones paralelas (Fig. 4).

La cara B presenta una serie de líneas incisas oblicuas que parten del extremo distal del soporte y convergen hacia el centro del mismo. Se pueden agrupar en dos

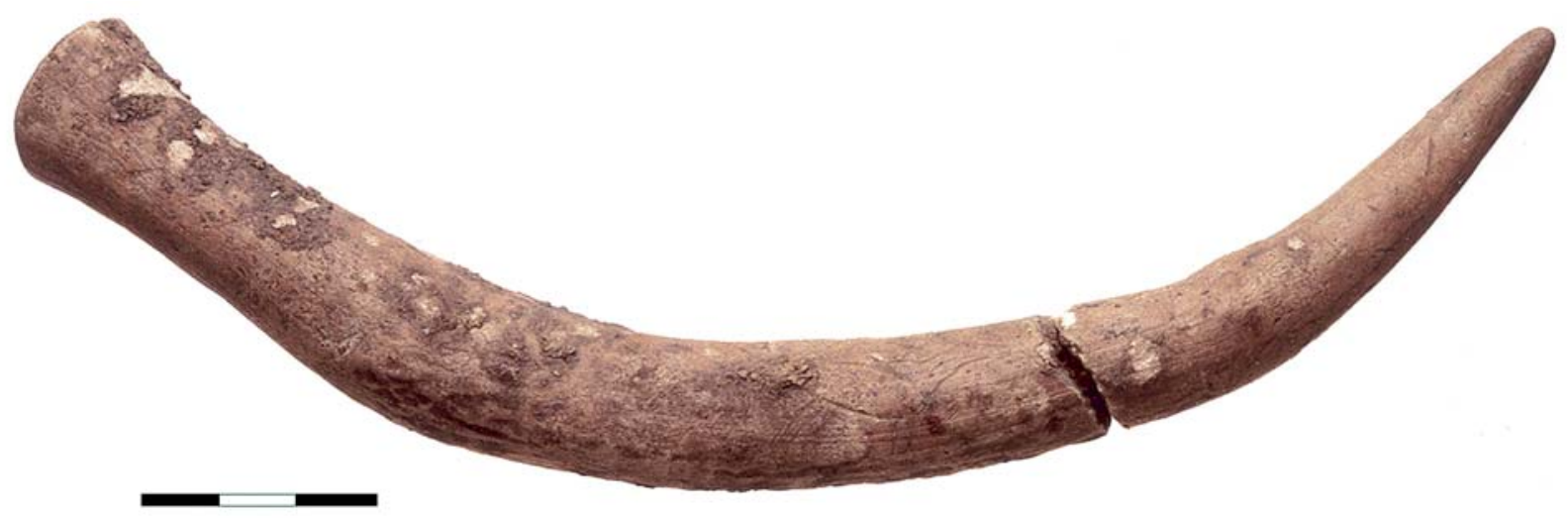

Fig. 1. Vista general de la pieza tal como se recuperó en la excavación / General view of the object as found in the excavation. 


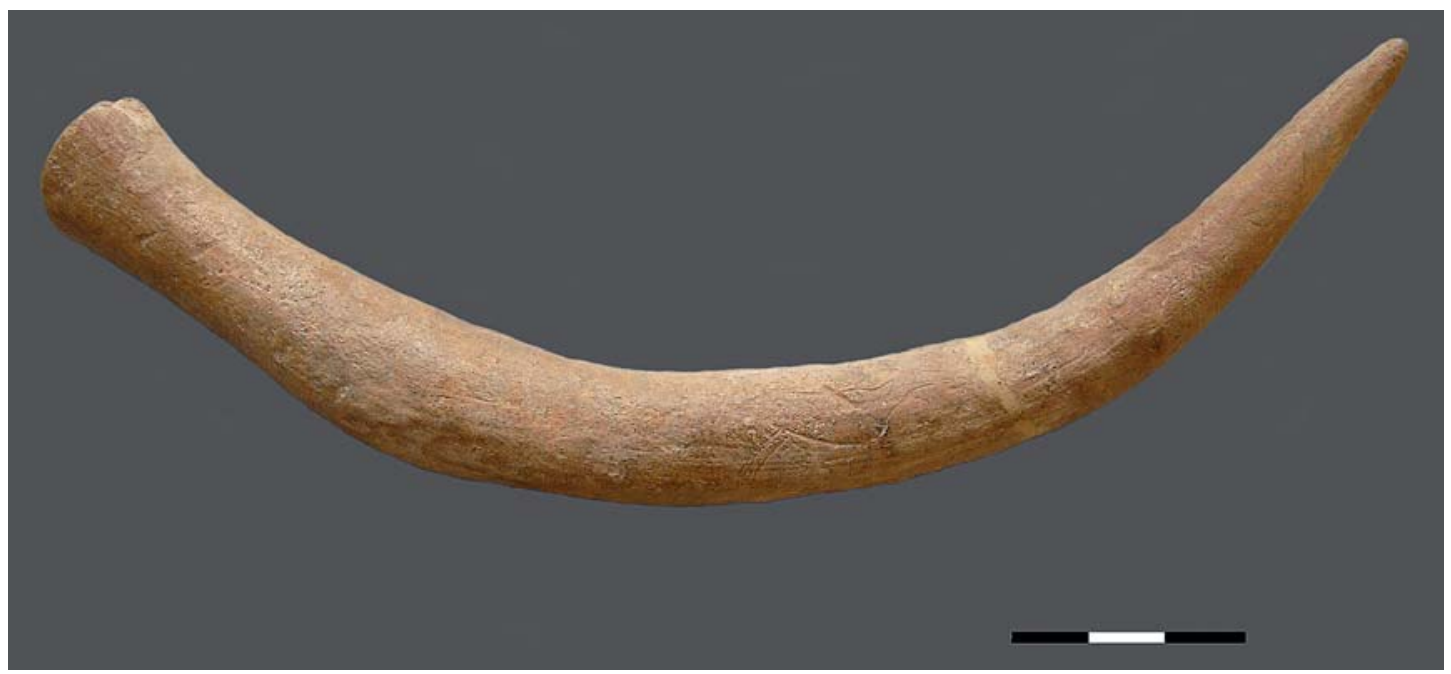

Fig. 2. Vista ge-

neral, cara A/Ge neral view, side $A$

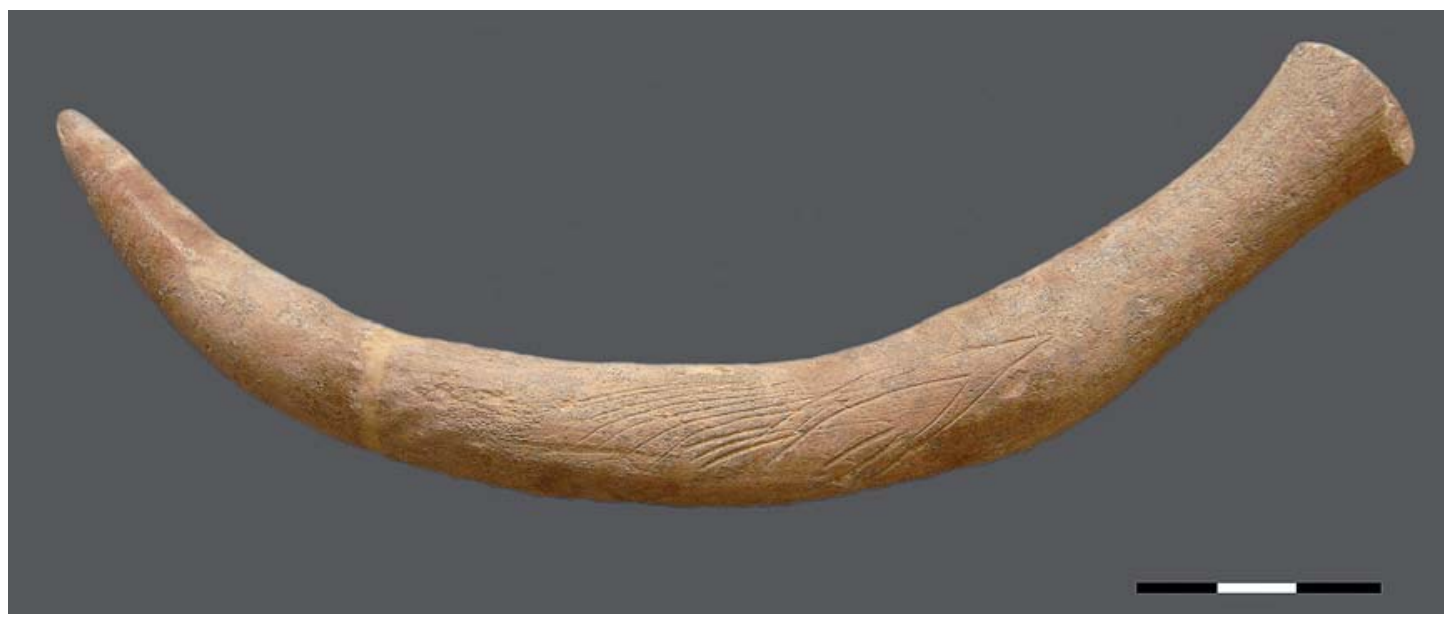

Fig. 3. Vista general, cara B / General view, side $B$.

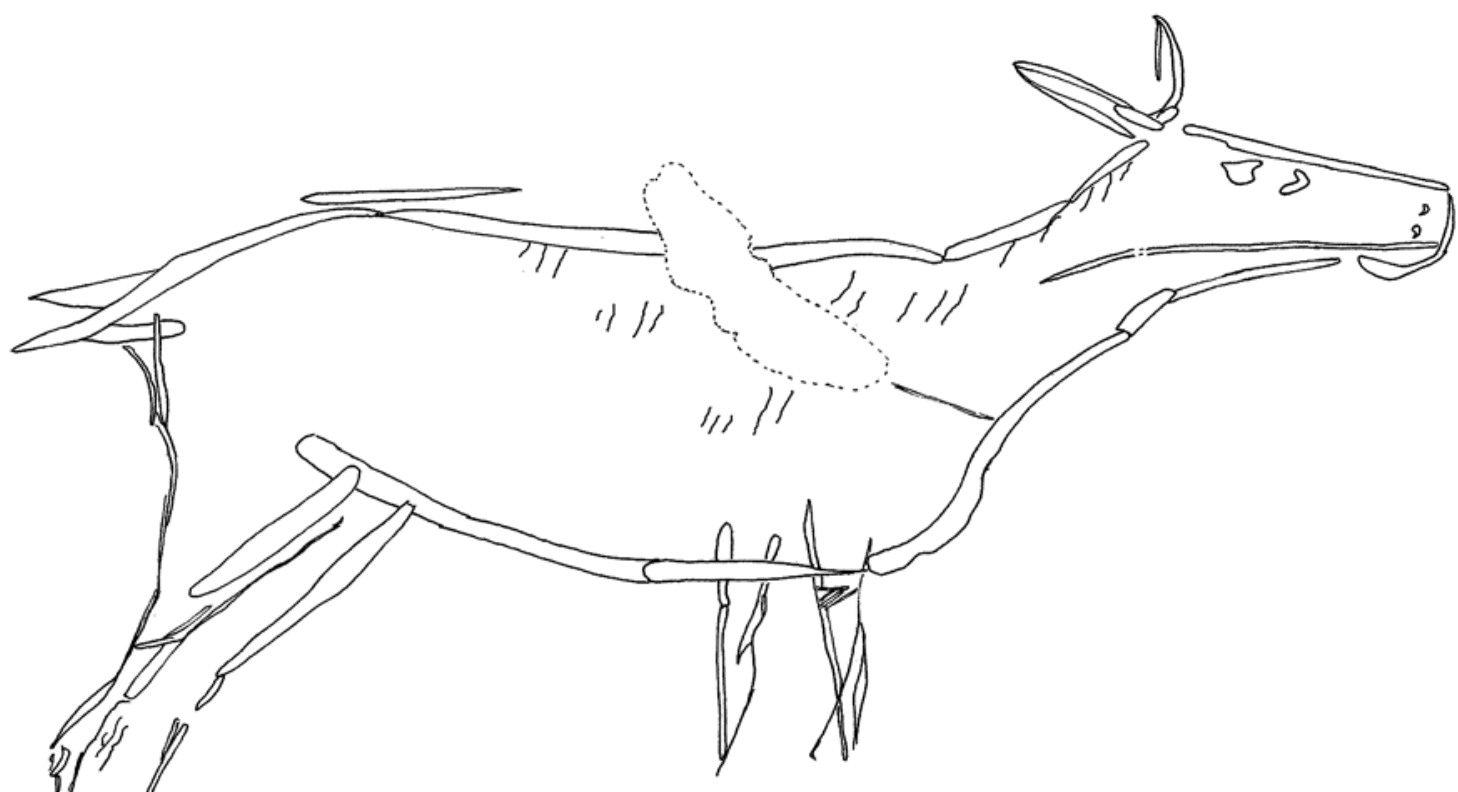

Fig. 4. Calco de la figura de la cierva / Tracing of the hind figure. 
conjuntos: el de la izquierda, en el que se identifican siete surcos convergentes y el de la derecha, compuesto de diez surcos subparalelos. Cuatro de los surcos del primer grupo se disponen en forma de dos $\mathrm{V}$ inscritas (Fig. 5).

\section{METODOLOGÍA}

La observación microscópica y la documentación gráfica que aportamos, fotos y calcos, se realizaron con una lupa Olympus SZX10 y el paquete informático asociado al propio equipo.

Llevamos a cabo un programa experimental adaptado al caso que estudiamos, lo que nos permitió reconocer aspectos relacionados con la obtención del soporte y sus distintas preparaciones, así como los de tipo tecnológico de los motivos grabados.

\section{OBSERVACIÓN MICROSCÓPICA DEL OBJETO}

\subsection{El soporte}

La totalidad de la superficie del soporte presenta un alisado que ha suprimido parcialmente las rugosidades naturales del asta. Este trabajo ha dejado líneas incisas no organizadas que se disponen longitudinalmente en la parte central y entre las que se observan algunos surcos profundos. Las rugosidades restantes y las líneas incisas se distribuyen de forma aleatoria sobre la superficie de la pieza y presentan un relieve de profundidad variable.

En el extremo distal observamos cuatro pequeñas incisiones no organizadas, perpendiculares al eje del soporte. De ellas, la más cercana al extremo apuntado tiene mayor profundidad y rodea un cuarto de su perímetro. Además tiene otras huellas superficiales que tampoco presentan orden (Fig. 6).

La zona proximal es convexa y tiene un acabado pulido. Este extremo forma una arista muy marcada y una parte de su contorno tiene un pequeño bisel. La arista muestra dos desconchados en partes opuestas, ambos son ovales y tienen un diámetro de unos $9 \mathrm{~mm}$. Uno de ellos tiene los bordes redondeados y pulidos mientras que el otro no presenta erosión ni pátina por lo que nos parece relativamente reciente (Fig.7)

En el tramo rebajado hay una serie de pequeñas incisiones perpendiculares al eje, de disposición subparalela y no colocadas de forma organizada (Fig.8).

La cara convexa del cuerpo central tiene un aplanamiento en el que se aprecian unas ondas, consecuencia de la acción de desbastado y raspado de la superficie. Todo el cuerpo central conserva áreas poco trabajadas, con irregularidades y rugosidades, que corresponden a la superficie natural del asta (Fig. 9)

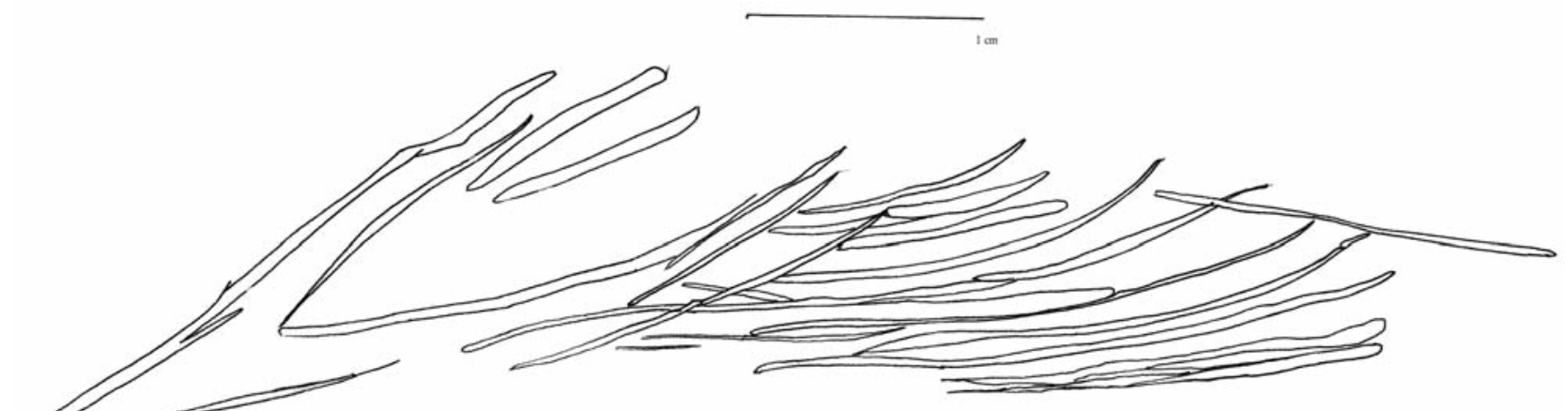

Fig. 5. Calco de la decoración de la cara B / Tracing of side B motif.

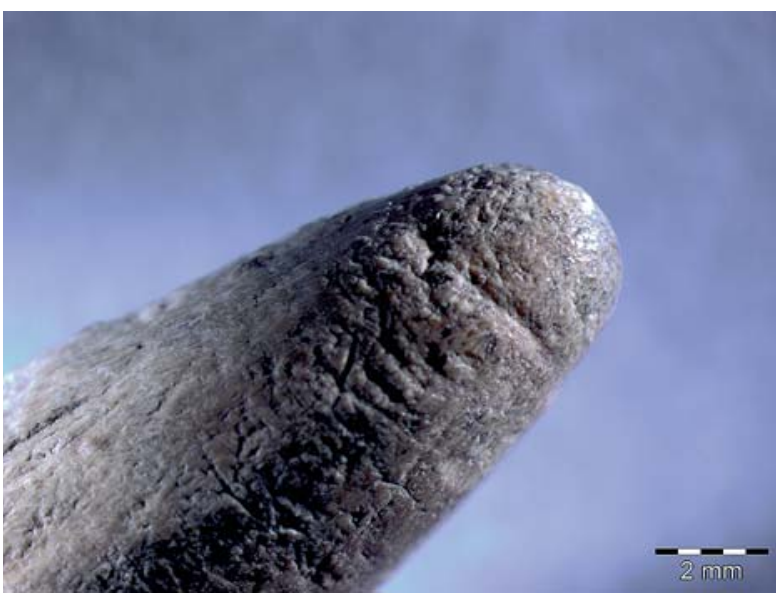

Fig. 6. Extremo distal / Distal end.

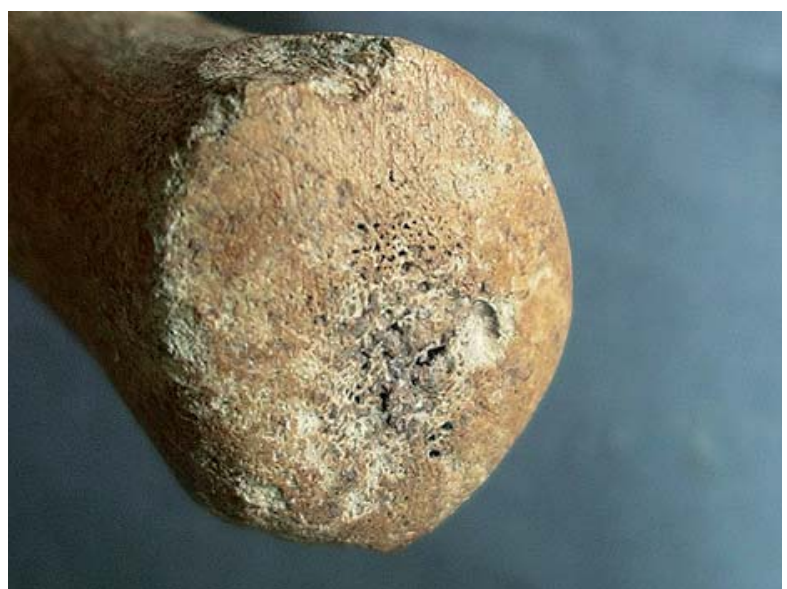

Fig. 7. Extremo proximal / Proximal end. 


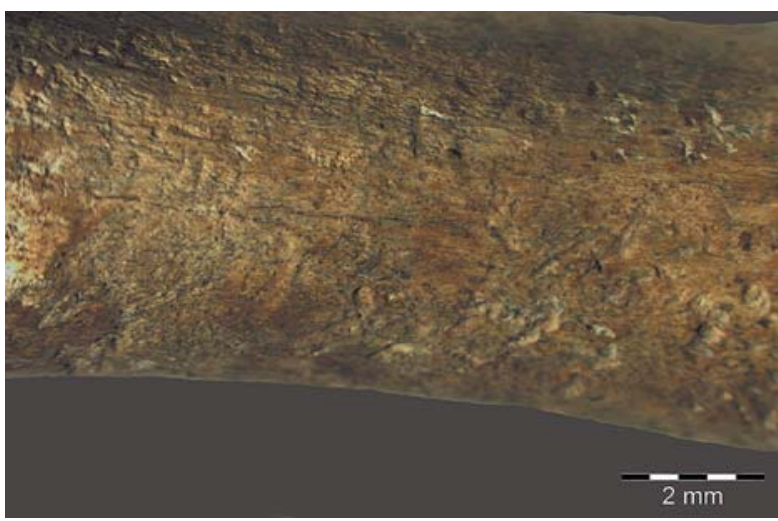

Fig. 8. Tramo rebajado en el que se pueden observar huellas transversales / Transverse traces on the lowered zone.

La pieza presenta un brillo similar al que hemos observado en las astas de ciervo. Es general en toda ella y más acusado en la punta y en el extremo proximal, aunque hay zonas que no lo tienen. Este hecho es igual al de las astas naturales.

\subsection{La decoración}

En lo que hemos denominado cara $A$, en la parte central del soporte y ligeramente desplazada hacia el extremo distal, se localiza una figura de cierva grabada. La cabeza se orienta hacia este extremo. La irregularidad de la superficie del asta en este punto es similar a la del resto de la pieza lo que nos indica que no ha existido una preparación especial.

El contorno de la figura está realizado por medio de un surco fino compuesto de pequeños trazos perfectamente encadenados de unos 4 o $5 \mathrm{~mm}$ de longitud. En lo que respecta a su profundidad hay diferencia entre los que delinean el pecho y la ingle y los de manos, patas, orejas y cola. Estos últimos son más superficiales. A pesar de que los surcos se encuentran muy erosionados se puede identificar en ellos una sección triangular (Fig.10).

El ojo, al igual que los dos ollares, se diseña con dos trazos en ángulo. La boca se destaca por medio del engrosa-

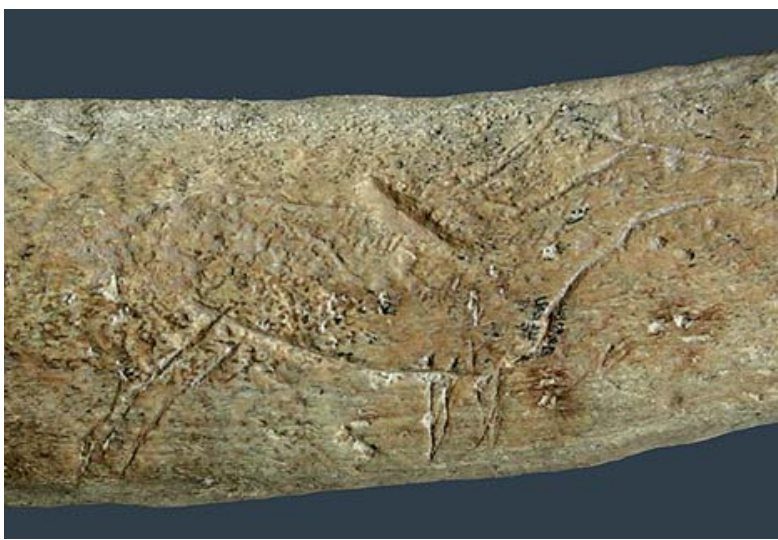

Fig. 10. Figura completa de la cierva / Full figure of the hind.

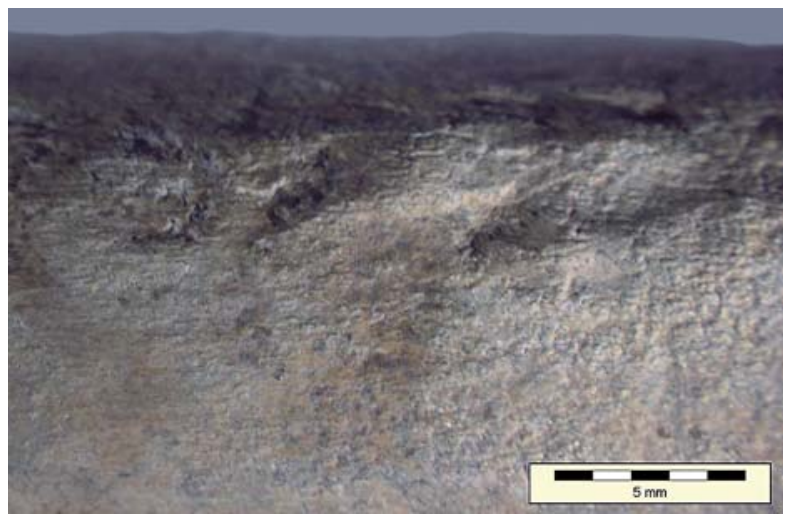

Fig. 9. Cara convexa de la zona central en la que se ven rugosidades / Rough zone on the convex face of the central part.

miento del surco. Una de las orejas se aprecia claramente debido a la profundidad del surco, mientras que la otra, más superficial, se identifica con más dificultad (Fig. 11).

El pelaje se dispone en la mitad superior del cuerpo, desde la cruz hasta la grupa. Algunos de los surcos están erosionados lo que dificulta reconocer su longitud y la forma de su sección. Su disposición es oblicua respecto al eje horizontal del animal. Son paralelos entre sí, están bien organizados y se sitúan a una distancia regular (Fig. 12).

La línea dorsal, inmediatamente después de la cruz del animal, tiene un desconchado muy erosionado lo que indica su antigüedad. En el interior del cuerpo de la figura encontramos otros levantamientos que guardan semejanza con los que aparecen en la superficie del soporte.

Las patas se han diseñado con varios trazos, que en su extremo inferior aparecen desflecados en varios haces (Fig. 13, 14 y 15).

Los surcos que componen la línea del pecho se montan ligeramente sobre el final del surco de la garganta, los de las manos se superponen a los del pecho, los de la línea dorsal sobre los de los cuartos traseros y éstos, sobre los de la panza.

A poca distancia del pecho de la cierva se observa un motivo, de 1,1 mm de longitud y 1,05 de anchura,

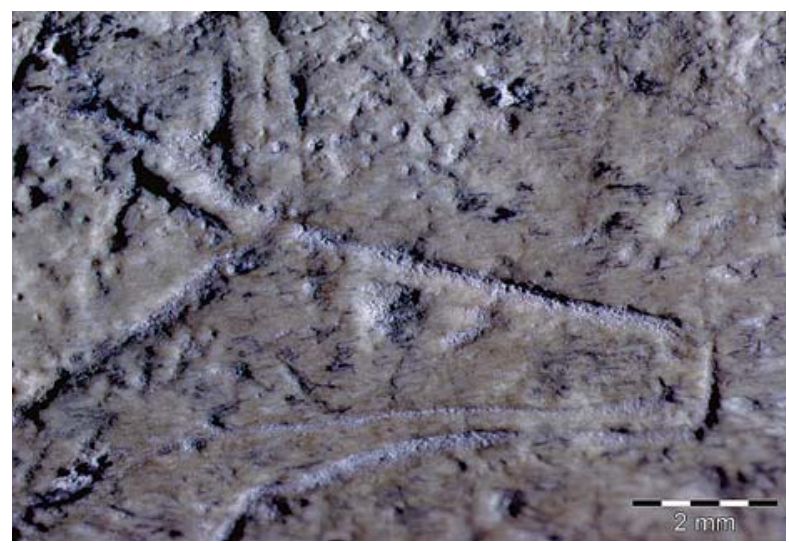

Fig. 11. Cabeza de la cierva / Head of the hind 


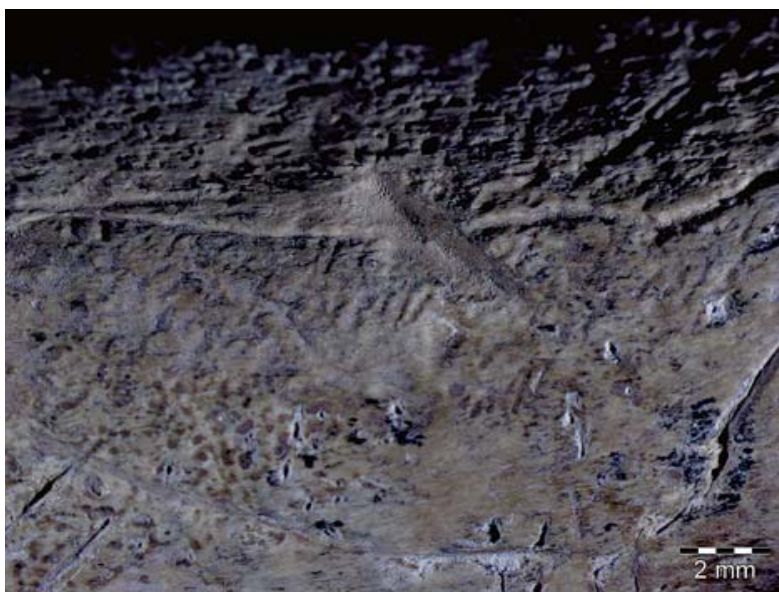

Fig. 12. Detalle del pelaje en el cuerpo de la cierva / Detail of coat on the hind's body.

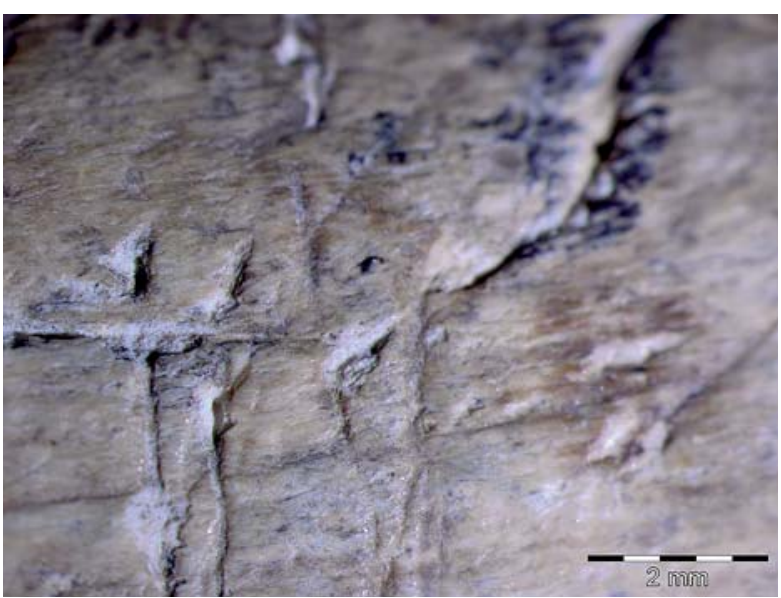

Fig. 14. Arranque superior de las manos / Upper start of fore-legs

compuesto de tres surcos paralelos y curvados, similares en dimensiones de longitud, anchura, profundidad y sección. Todos ellos presentan un trayecto de izquierda a derecha. El superior se ha incurvado, en un repasado posterior, hasta unirse con el inferior. A poca distancia del surco central, se sitúa otro con parecida trayectoria, dimensiones y sección pero más erosionado (Fig.16).

En el espacio situado por encima de la cabeza se disponen unos surcos de inclinación variable de los cuales uno se acerca a la cruz del animal. El hecho de que no entren en contacto con el contorno de la figura no nos permite decir que haya relación entre ellos.

En el grabado de la cara B podemos determinar, a efectos descriptivos, dos zonas. La izquierda se compone de dos surcos de trayecto suavemente curvado, orientados hacia el extremo proximal del objeto, que tienden a converger en su extremo izquierdo. Estos surcos inscriben otros dos, de menor longitud, que repiten un poco el diseño anterior: son también convergentes y tienen la misma orientación y dimensiones de anchura y profundidad (Fig. 17 y 18).

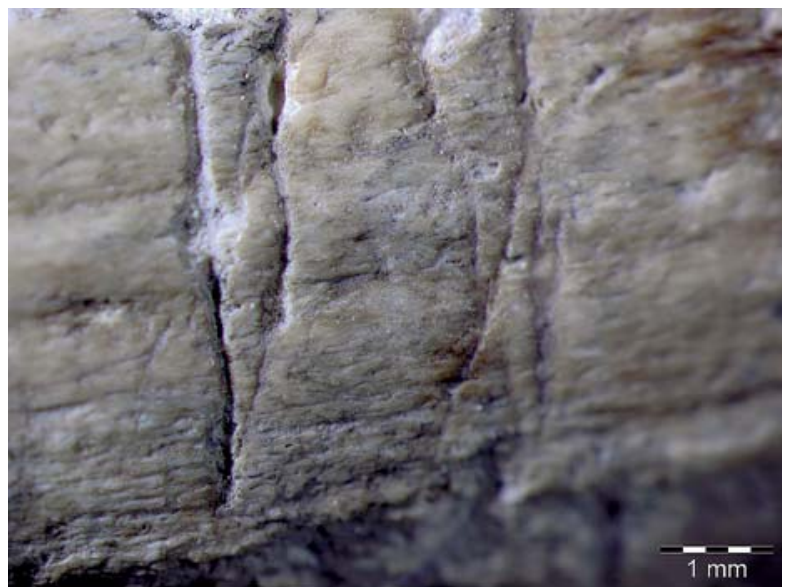

Fig. 13. Parte inferior desflecada de las manos / Lower part of frayed fore-legs.

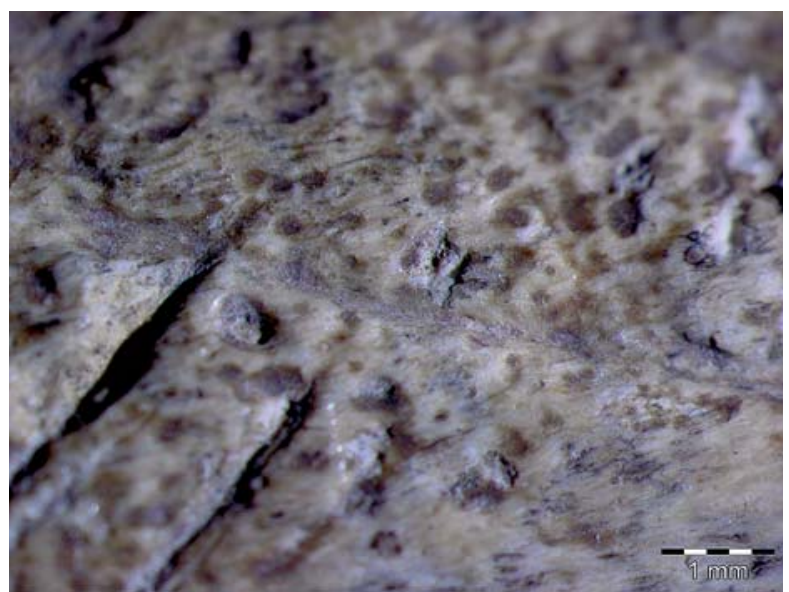

Fig. 15. Arranque superior de las patas / Upper start of hind legs.

Superpuesto en parte al surco superior se han grabado tres surcos paralelos, muy cortos, más profundos que los anteriores y de trayectoria ligeramente curvada. Uno de ellos parece una prolongación del surco exterior del motivo descrito.

El grabado de la zona derecha se compone de 10 surcos subparalelos, ligeramente curvos que divergen en el extremo derecho. Los siete inferiores se han grabado de izquierda a derecha. Los tres superiores son más anchos, más cortos y más profundos y además, están hechos en sentido contrario a los anteriores (Fig. 19).

Ambos grupos de surcos tienen algunas características formales que los hacen semejantes: exactitud y regularidad de los trazos y perfecta conexión de los ángulos que los unen.

\section{PROGRAMA EXPERIMENTAL}

Como soporte para nuestra experimentación empleamos una cuerna de ciervo que nos proporcionó amablemente el responsable de El Carpín (Bizkaia) 


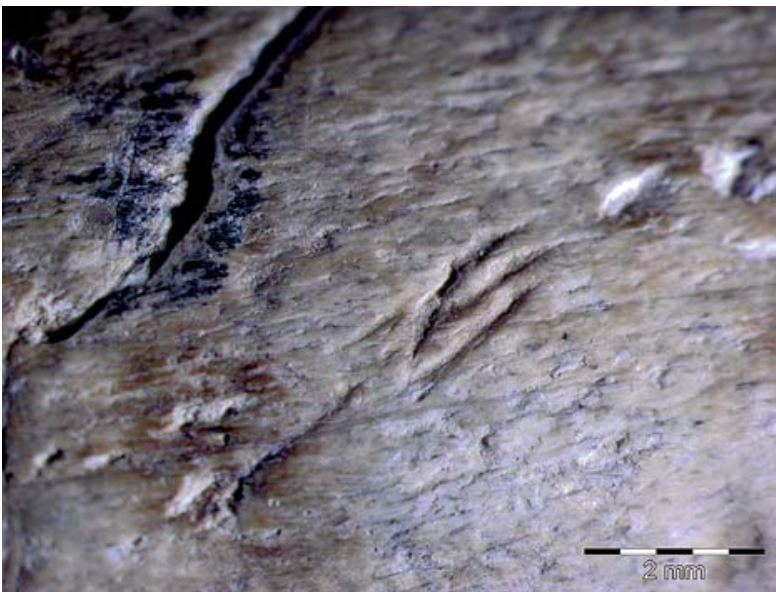

Fig. 16. Signo situado delante del pecho de la cierva / Sign in front of the hind's chest.
Pedro Abad. Este soporte se utilizó humedecido y seco en todos los trabajos y no se observaron diferencias en los resultados. Tras la observación macroscópica del objeto arqueológico intentamos reproducir las modificaciones que había sufrido.

En primer lugar procedimos al serrado de algunas puntas con el fin de lograr piezas de un grosor, longitud y forma similares a la arqueológica. A continuación, realizamos un trabajo de redondeamiento del extremo proximal y de la arista con una piedra arenisca.

Posteriormente raspamos y cortamos la superficie con el filo de una lámina de sílex, con un movimiento similar al que se haría con una navaja, a fin de eliminar las rugosidades. Esta operación se realizó con facilidad por medio del raspado con un sílex quedando huellas de las zonas más profundas de la topografía del asta,

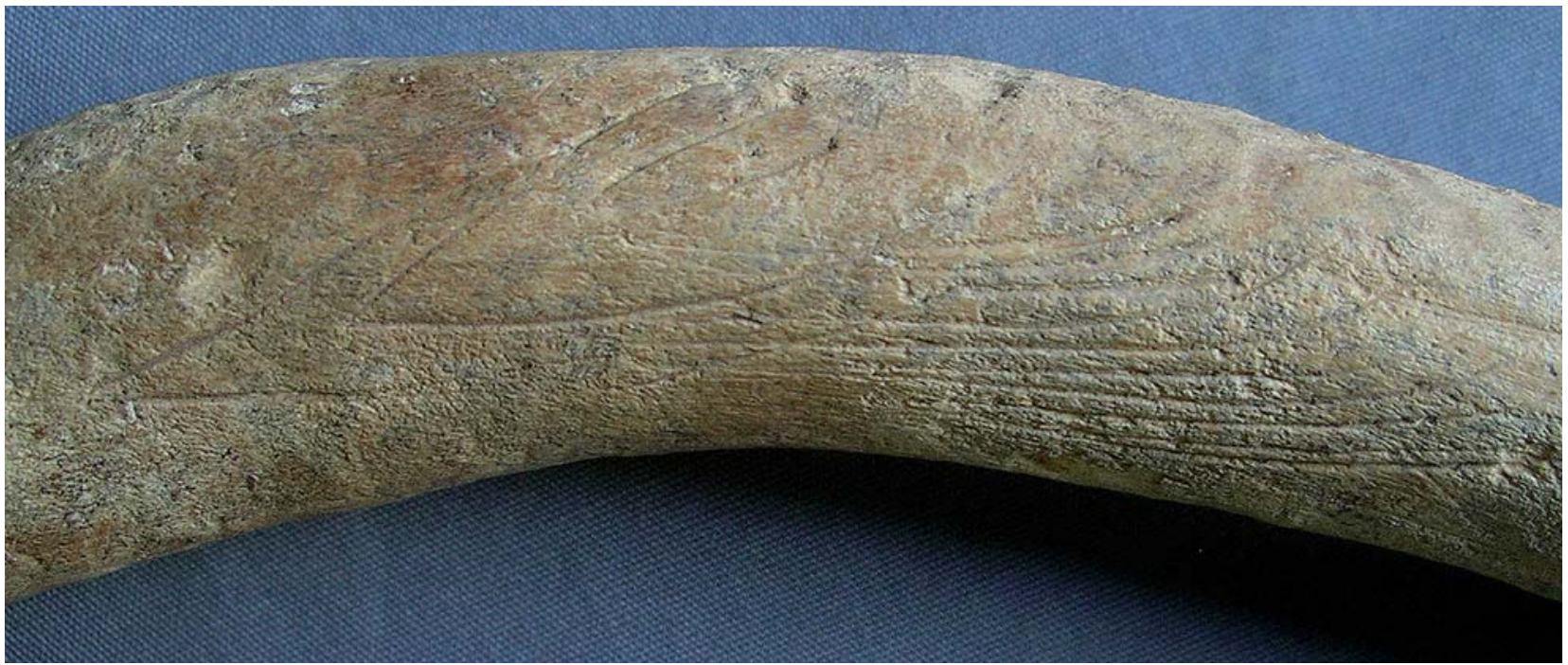

Fig. 17. Motivo decorativo completo de la cara B / Full decorative motif on side B.

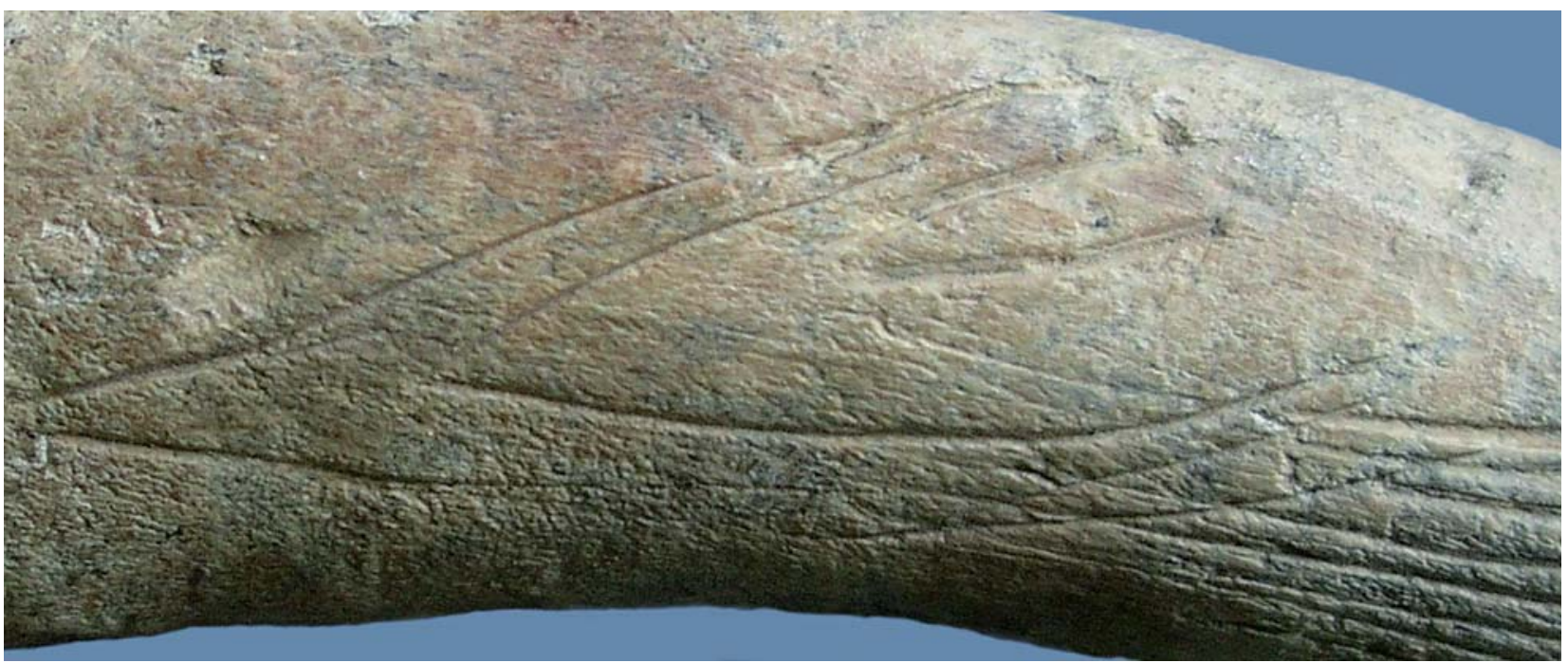

Fig. 18. Grupo izquierdo del motivo de la cara B / Left group of motif on side B. 


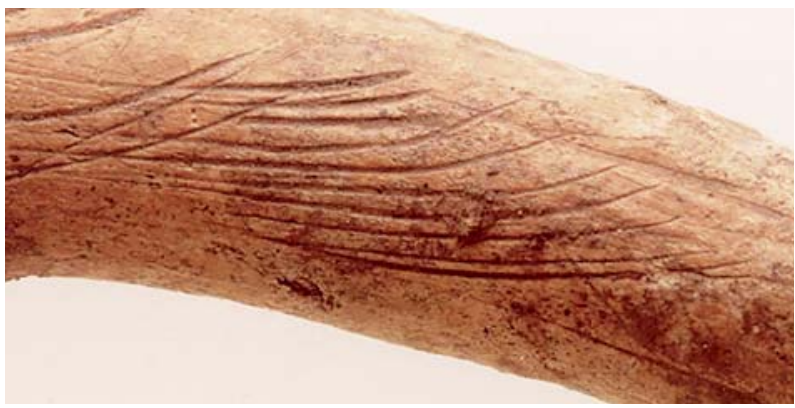

Fig. 19. Grupo derecho del motivo de la cara $B$ / Right group of motif on side $A$.

tanto de las zonas más deprimidas como de los surcos naturales (Fig 20).

El rehundimiento que encontramos en el tramo rebajado, cercano a la parte proximal del objeto arqueológico, se obtuvo de la misma manera antes descrita para el desbastado de la superficie hasta lograr la profundidad y forma deseada (Fig. 21).

En lo relativo a la decoración han hecho los surcos grabados en ambas caras de una única pasada, sin producirse un repasado posterior. La conexión de los trazos que componen un surco, así como las conexiones en ángulo, se habían realizado de forma precisa. No encontramos dificultad en llevar a cabo estas tareas utilizando, como así lo indica la forma triangular de la sección del surco, el ángulo triedro de un buril. Las diferentes profundidades observadas en distintas zonas de la figura se han producido al ejercer una presión distinta en el grabado. Los detalles de orejas, ojos y ollares han requerido de una punta fina. Por último, en efecto, se observa que la redondez del soporte puede ocasionar que las patas se terminen de forma no cuidada, con un desflecado de trazos.

\section{RESULTADOS}

La preparación a la que se sometió el objeto antes de ser decorado fue el desbastado de las rugosidades. De esta primera operación tenemos restos del fondo de los

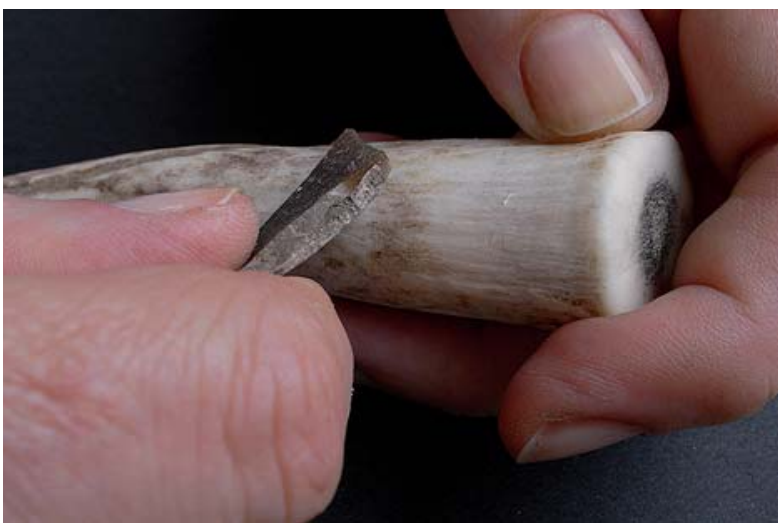

Fig. 20. Raspado experimental de la superficie del asta / Experimenta scraping of antler surface. surcos naturales del asta más profundos, así como otros surcos más superficiales en forma de líneas no organizadas que afectan a gran parte de la superficie. Las labores de desbastado de la superficie han podido ser realizadas por medio de una lámina o lasca con filo.

Estas mismas huellas de raspado — haces de líneas incisas longitudinales pero no organizadas - las encontramos claramente en el tramo rebajado, cercano a la zona proximal. En ésta observamos algunas líneas finas, transversales al eje de la pieza que no creemos que puedan responder a las labores de preparación sino a su uso.

Tanto el tramo rebajado como la forma redondeada de la parte proximal del objeto se han trabajado de manera concienzuda. Esta forma redondeada, con huellas de líneas finas posicionadas en distintas direcciones, ha podido obtenerse por raspado sobre una superficie rugosa. Estas huellas así como la presencia de una zona del extremo proximal bien delimitada más desgastada que el resto de la superficie pueden explicarse bien por el proceso de fabricación, bien por una erosión debida al uso. No es posible reconocer la intención de los levantamientos de materia presentes en su arista.

Las huellas que observamos en el extremo distal del objeto arqueológico, su escasa representación y lo superficial de las mismas, nos indican que no pueden ser consecuencia de un trabajo sistemático ni específico. Los levantamientos en la cruz del animal y en el interior de la figura, así como otros localizados en el resto del soporte son de aspecto postdeposicional.

En lo relativo a la decoración hemos podido deducir que todas las representaciones son producto de una sola pasada del instrumento, que en ambas caras del objeto las conexiones de los surcos se han hecho de forma precisa, que el triedro de un buril o lámina robusta es la parte activa que ha producido la sección triangular de todos los surcos - salvo los muy superficiales o los pocos que se encuentran excesivamente erosionados-, que la longitud de los trazos que componen los contornos o líneas principales son similares y que las otras dimensiones de anchura y profundidad son variables dentro de la misma

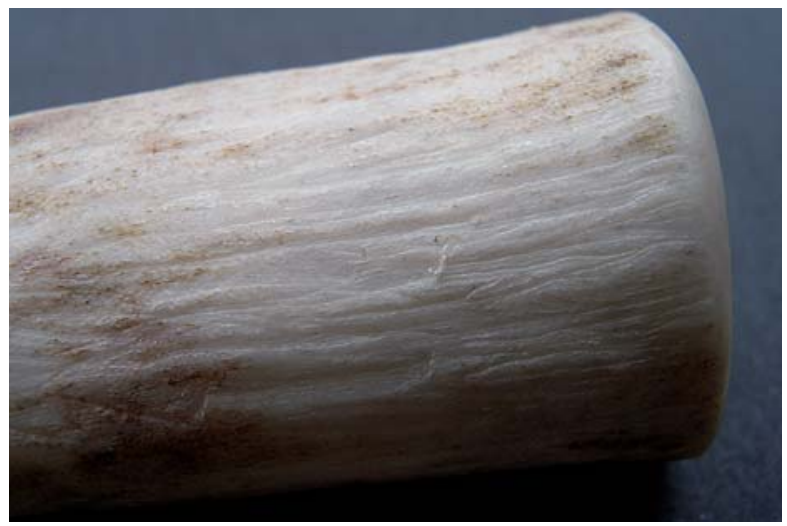

Fig. 21. Huellas experimentales del raspado / Traces of experimental scraping 
figura, pero parecidas en los surcos de todas las formas decorativas del objeto.

En la figura de cierva la cabeza y garganta se han grabado en primer lugar y las manos antes que la línea del pecho. La línea dorsal se ha realizado antes que los cuartos traseros y éstos, tras la línea ventral. Es decir que el autor trazó la figura de derecha a izquierda.

Las patas, en su extremo inferior, en el punto en el que coinciden con la curvatura del soporte, terminan en unos trazos desflecados en varios haces.

En la cara B, las líneas incisas de la zona derecha se superponen a las de la izquierda y por lo tanto fueron realizadas en este orden.

Ambos grupos de surcos tienen características formales que los hacen semejantes. La meticulosidad del diseño: exactitud, regularidad de los trazos, seguridad de la mano en la delineación de los surcos y perfecta conexión de los ángulos, y la semejanza de los dos motivos, tanto por las dimensiones y forma de los surcos como en el hecho de que ambos llevan asociado un motivo compuesto de tres surcos, nos llevan a pensar que han sido ejecutados por una misma mano.

Finalmente hay que señalar que se ha transformado la forma del soporte natural exclusivamente en lo que hemos denominado extremo proximal y tramo rebajado y no observamos huellas de entidad en el resto del soporte. Nuestra experimentación muestra que el redondeamiento de la parte proximal deja huellas desorganizadas y no muy abundantes en la superficie, similares a las observadas en esta parte de la pieza arqueológica.

En el tramo rebajado hay un único levantamiento de aspecto antiguo y hemos descrito unas líneas pequeñas muy finas perpendiculares al eje del soporte. Estos estigmas, escasos y poco profundos, unidos a la poca longitud de este tramo - no de amplitud suficiente para ser asido con la mano-, nos hacen pensar en un objeto que no fue usado como herramienta y en que esta parte pudo tener relación con su enmangamiento o con un sistema de sujeción.

\section{PARALELOS}

No hemos encontrado ninguna pieza semejante a la de Santa Catalina en la Península. Sin embargo, algunas recuperadas en yacimientos del Pirineo francés presentan algunas similitudes con ella. Se trata de dos objetos de arte mueble decorados de la cueva de La Vache denominados «poignard» (WELTÉ, 2003. WELTÉ y ROBERT, 1993) y «sceptre» (NOUGIER y ROBERT, 1976; DELPORTE, 2003) y uno, sin decoración, de la de Isturitz' (Fig 22).

Las dos primeras se recuperaron en niveles diferentes de la denominada Salle Monique. El «poignard» en la "couche" 2 y el «sceptre» en la "couche" 4. Ambos niveles están atribuidos al Magdaleniense final. La pieza de Isturitz se encontró en el nivel I atribuido al Magdaleniense superior.

Todos los ejemplares franceses están hechos en candil de ciervo y tienen una morfología general similar. Después de retirar total o parcialmente la superficie rugosa natural, en la parte contraria al ápice del candil se ha redondeado el extremo proximal y se ha rebajado la materia de la parte próxima hasta obtener un perfil cóncavo en todo su derredor. Las dimensiones de los dos ejemplares decorados de La Vache son parecidas a las del de Santa Catalina, 293 mm el «poignard» y 210 el «sceptre». Por el contrario, la pieza de Isturitz mide solo $130 \mathrm{~mm}$.

En ninguno de los casos que nos ocupan se han hecho análisis tecnológicos detallados sobre su manufactura y posible funcionalidad. El nombre con el que se conocen las piezas de La Vache, hace pensar en una relación formal con un posible uso puesto que el nombre de puñal no está apoyado en el hecho de que haya señales de haber servido para ese uso, al menos no se mencionan, y el de cetro únicamente quiere señalar una interpretación como elemento ritual, aunque también se apunta la posibilidad de que fuera enmangado en el extremo de una soporte mayor (NOUGIER y ROBERT, 1976). La presencia de un rebaje cóncavo en ambas piezas ha hecho suponer que hubieran servido para ser empuñadas (WELTÉ, 2003). En el caso de Isturitz, J.A. Mujika interpretó la presencia de unas huellas que han desgastado el extremo proximal como señales de su empleo como bruñidor.

La decoración en las dos piezas de La Vache es muy abigarrada y tiende a ocupar toda la superficie. En ambas, el elemento decorativo principal es de carácter animalístico: ciervos, cabra y posible pez en una de ellas y un conjunto más variado en la segunda: pez, cérvido, felino, caballo y una cabeza de ave. La técnica empleada es completamente diferente en ambas puesto que en el «poignard» las representaciones están únicamente incisas mientras que en el «sceptre» son decoraciones en relieve.

Ambos objetos se interpretan como cargados de un fuerte carácter simbólico. El «poignard» no solo acumula alrededor de una figura poderosa de ciervo un conjunto de animales esquemáticos y otras representaciones de difícil interpretación, sino que su extremo agudo ha sido trabajado en forma de pene.

En el caso de Santa Catalina la representación figurativa de la cierva aparece aislada.

La decoración de la cara B que aparentemente no tiene carácter figurativo, sin embargo, recuerda al esquematismo que suele emplearse para la representación de las cuernas de los cérvidos. Esto es más evidente en el grupo de trazos de la izquierda.

\footnotetext{
${ }^{1}$ Comunicación personal de J.A. Mujika, quien nos ha proporcionado tanto la descripción como la representación gráfica de la pieza.
} 

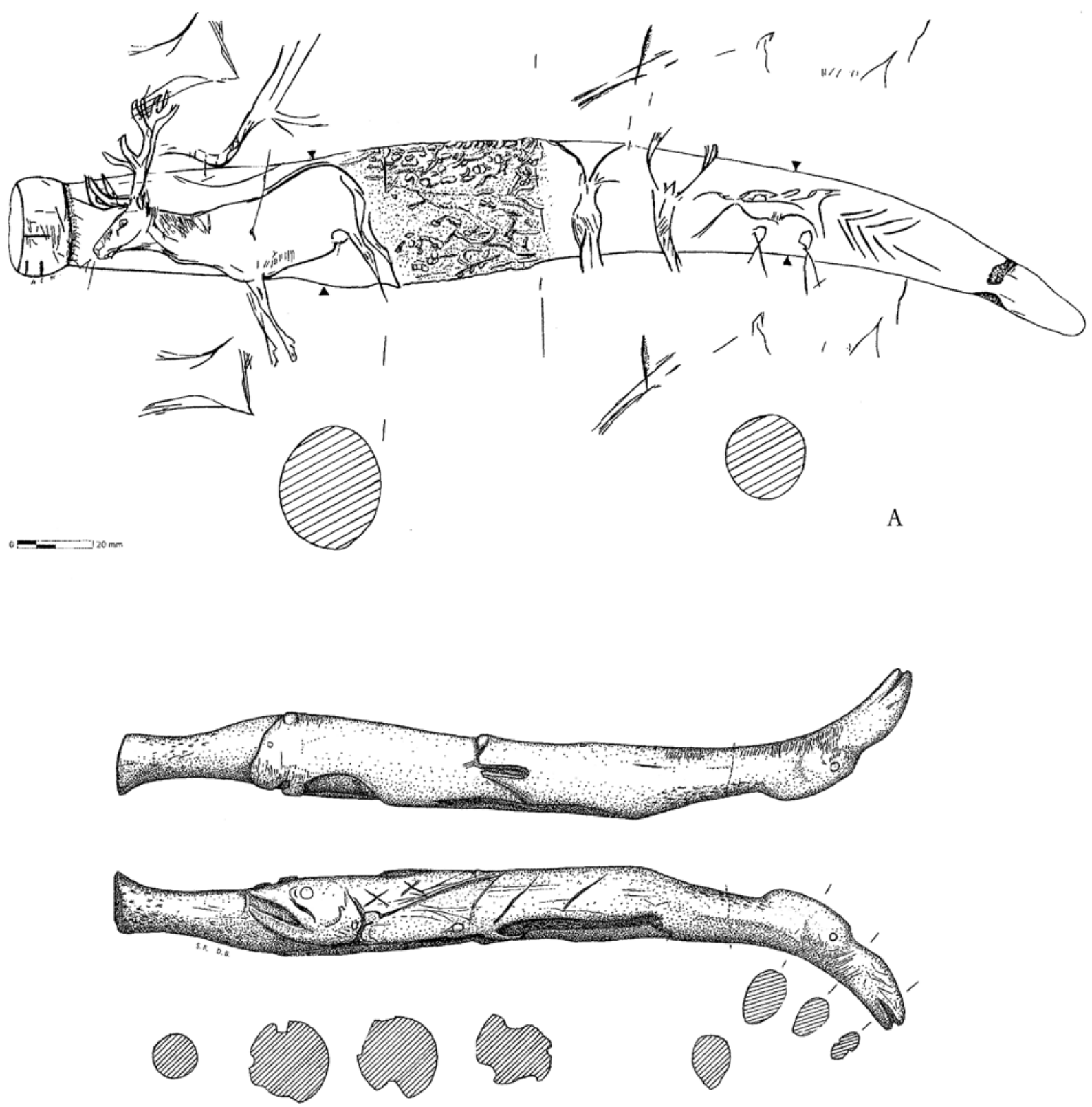

B

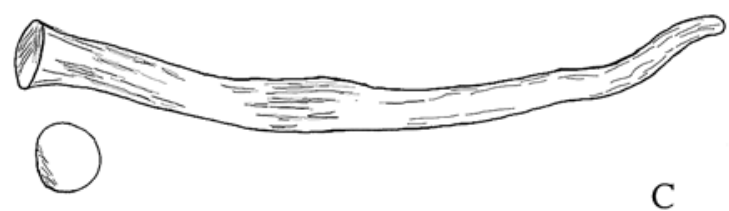

Fig. 22. A "Le poignard" de la cueva de La Vache (según A.C.Welté), B "Le sceptre" de la cueva de La Vache (según S.R. y D.Buisson) y C candil de ciervo trabajado de la cueva de Isturitz (según J.A.Mujika) / A "Le poignard" from La Vache cave (according to A.C.Welté), B "Le sceptre" from La Vache cave (according to S.R. and D. Buisson) and C carved upper side of deer antler from Isturitz cave (according to J.A. Mujika). 
Algunas representaciones de cornamentas tanto de renos como de ciervos suelen tener unos trazos en forma de $\mathrm{V}$ inscritos unos en otros, a los que se añaden más o menos elementos para figurar los candiles. Esto lo encontramos en cuevas del Cantábrico como El Castillo, Las Chimeneas, Santimamiñe, etc.

No puede descartarse que también en este caso se tratara de una figuración esquematizada de la cornamenta de un cérvido. No es fácil poder distinguir si se trata de un ciervo o un reno. No habría que desechar que lo mismo ocurriera con el conjunto de la derecha en el que aparecen una serie de trazos paralelos.

Por otra parte, la decoración que hemos descrito como grupo de la derecha de la cara B es posible que forme parte junto con la de la izquierda de una misma composición

\section{CONCLUSIONES}

La pieza que estudiamos se ha realizado sobre una punta de asta de ciervo. Esta punta se ha separado del asta por un serrado y, posteriormente, se ha redondeado en uno de sus extremo proximal. En el tramo cercano a esta parte se ha realizado un rebaje en todo el contorno. Hemos podido determinar asimismo que la superficie del soporte ha sufrido, de forma previa a ser decorada, un desbastado de las rugosidades y un alisado que ha dejado sus huellas en la superficie.

En la punta no observamos ningún vestigio de preparación. Sí encontramos en esta zona unas pequeñas incisiones que circunvalan parcialmente el objeto. Sin embargo, no nos parecen responder ni a un uso ni a una decoración precisa o terminada.

Creemos que las huellas y el redondeamiento de la parte proximal tuvieron su origen en la operación de dar forma al objeto puesto que la intensidad de las huellas —al menos las que pueden ser ahora apreciadas_, es escasa para que pudiera tratarse de un uso prolongado, acorde con una herramienta destinada a transformar otra materia.

El tramo rebajado no tiene una amplitud tal que permita ser asido con la mano. Por otra parte, hemos descrito unos levantamientos de materia presentes en la arista de la zona proximal y unas líneas transversales que no parecen responder a labores de preparación del soporte. Estas modificaciones sugieren que la zona pudo estar enmangada. Sin embargo, la ausencia de otros estigmas no nos permite plantear con seguridad esta interpretación.

En ninguna otra parte del soporte: punta, parte proximal, tramo rebajado o cuerpo del objeto apreciamos una alteración uniforme o de concentración de huellas que nos hagan pensar en este objeto como un útil destinado a modificar otra materia, como pudieran ser huellas de fricción, percusión, etc

La decoración de la cara $A$ se reduce a una figura grabada de cierva. En ella se ha diseñado cuidadosa- mente el contorno de la cabeza, la línea anatómica dorsal y la ventral, las patas y manos. Se han incluido detalles anatómicos precisos como los ojos, ollares, boca, orejas, cola y pelaje.

En la cara B se disponen dos grupos de surcos. En ambos los surcos se colocan a distancias regulares, las conexiones de trazos se realizan con precisión y las dimensiones de profundidad y anchura son muy regulares.

No resulta fácil saber qué puede ser lo representado en estos dos conjuntos casi idénticos de la cara B pero la precisión de su factura nos hace pensar que tiene una intención representativa e incluso figurativa. El hecho de que se trate de una punta de asta de ciervo y que sea una cierva la figura representada en la cara $A$, nos ha sugerido, con todas las reservas, que pueda tratarse de una representación esquemática o expresionista de un asta de ciervo.

Comparando la decoración de ambas caras vemos que el trazado es de una única pasada y que los surcos de la cara B son más profundos y anchos que los de la figura de la cierva. En las dos el grabado se ha realizado por medio del triedro de un buril o lámina fuerte y son semejantes entre sí en algunos aspectos como las dimensiones de longitud de los trazos, la forma de los surcos y también, de forma destacada, en la precisión del trazado. No lo son, sin embargo, en la dirección de los trazos y nada sabemos tampoco de la semejanza entre ambas caras desde el punto de vista del orden de realización del motivo.

Poco podemos decir de los motivos de tres surcos presentes en las cercanías tanto de la figura de cierva como del grabado de la cara $\mathrm{B}$. El hecho de que no haya más motivos de este tipo en el resto del soporte así como su repetición en unión de las representaciones principales, nos lleva a pensar que están relacionadas, al tiempo que sugiere la unidad representativa de ambas caras del objeto.

Las razones anteriormente señaladas nos llevan a considerar que se trata de un conjunto único que pudiera ser la obra del mismo artista.

Habría que plantear una hipótesis sobre el posible significado de esta pieza. En primer lugar, sabemos que hemos encontrado huellas consecuentes a la modificación de la forma original del soporte y otras que hemos atribuido a un posible enmangado o sujeción.

En segundo lugar, la decoración descrita sitúa una figura de cierva en una cara y, en la otra, una representación que hemos interpretado como un asta de cérvido. Nuestra dificultad para conocer la cosmovisión de estas poblaciones humanas nos impide saber la intención del artista. Sin embargo, algunos rasgos como el tratarse de una pieza singular, lo elaborado del soporte, los pocos elementos representados en la decoración, y la importancia de cada figura, el detallismo de los contornos y detalles anatómicos, las conexiones de los trazos, etc., nos llevan a pensar que se trata de unos elementos de- 
corativos buscados intencionalmente y que no se deben a un simple azar. Por otra parte, en el mismo yacimiento de Santa Catalina se encontró un colgante en hueso decorado, en el que establecimos una relación entre la representación de un uro y una combinación de motivos de tipo esquemático que podía interpretarse como un símbolo femenino (BERGANZA y RUIZ IDARRAGA, 2002; RUIZ IDARRAGA y BERGANZA, 2012).

Creemos que esta pieza tiene un carácter singular con un significado especial. Posiblemente se trataría de un objeto de prestigio o que tuviera un uso ritual.

\section{BIBLIOGRAFÍA}

BERGANZA, E., RUIZ IDARRAGA, R.

2002 Un colgante decorado magdaleniense del yacimiento de Santa Catalina (Lekeitio, Bizkaia). Munibe 54, 67-77.

BERGANZA, E.; ARRIBAS, J. L.; CASTAÑOS, P.; ELORZA, M.; GONZÁLEZ URQUIJO, J. E.; IBÁÑEZ, J. J.; IRIARTE, M. J.; MORALES, A.; PEMÁN, E.: ROSALES, T.; ROSELLÓ, E.; RUIZ IDARRAGA, R.; URIZ, A.; UZQUIANO, P.; VÁSQUEZ, V. y ZAPATA, L.

2012 La transición tardiglaciar en la costa oriental de Bizkaia: el yacimiento de Santa Catalina. Resultados preliminares. En ARIAS CABAL, P.; CORCHÓN RODRÍGUEZ, M.S.; MENÉNDEZ FERNÁNDEZ, M. y RODRÍGUEZ ASENSIO, J.A. El Paleolítico Superior Cantábrico. Actas de la primera mesa redonda. San Román de Candamo (Asturias) 26-28 de abril de 2007. 171- 182. Santander. Universidad de Cantabria.

DELPORTE, $\mathrm{H}$.

2003 Bois de cervidé dit «Le Sceptre». En CLOTTES, J., DELPORTE, H. (eds.). La grotte de La Vache (Ariège) fouilles Romain Robert. II-L'àrt mobilier, 404-405.

NOUGIER, L.R., ROBERT, R.

1976 «Sceptre» du Magdalénien final pyrénéen. Prehistoire ariégeoise 31, 55-61.

RUIZ IDARRAGA, R., BERGANZA, E.

2012 Un colgante magdaleniense de la cueva de Santa Catalina. Análisis tecnológico y funcional. Isturitz, 12, 61-81.

WELTÉ, A.C

2003 Bois de cervidé dit «Le poignard». En CLOTTES, J., DELPORTE, H. (eds.) La grotte de La Vache (Ariège) fouilles Romain Robert. II-L’àrt mobilier, 400-401.

WELTÉ, A.C.; ROBERT, R.

1993 Le «poignard» (Coll. R. Robert) de la grotte de La Vache, à Alliat (Ariège): Contribution à l'étude du décor au Magdalénien final. B.S.P.F. 90, 219-228. 